\title{
The Degree of Mutual Anisotropy of Biological Liquid Crystals Net during the Diagnostics of Human Tissues Birefringence
}

\author{
Oleg V. Angelsky and Yuriy A. Ushenko \\ Correlation Optics Department, Chernivtsi National University, 2 Kotsyubinsky Str., 58012 Chernivtsi, Ukraine \\ Correspondence should be addressed to Yuriy A. Ushenko, o.ushenko@chnu.edu.ua
}

Received 1 December 2009; Accepted 6 April 2010

Academic Editor: Igor I. Mokhun

Copyright ( $) 2010$ O. V. Angelsky and Y. A. Ushenko. This is an open access article distributed under the Creative Commons Attribution License, which permits unrestricted use, distribution, and reproduction in any medium, provided the original work is properly cited.

To characterize the degree of consistency of parameters of the optically uniaxial birefringent liquid crystals (protein fibrils) nets of biological tissues a new parameter-complex degree of mutual anisotropy is suggested. The technique of polarization measuring the coordinate distributions of the complex degree of mutual anisotropy of biological tissues is developed. It is shown that statistic approach to the analysis of complex degree of mutual anisotropy distributions of biological tissues of various morphological and physiological states and optical thicknesses appears to be more sensitive and efficient in differentiation of physiological state in comparison with investigations of complex degree of mutual polarization of the corresponding laser images.

\section{Introduction}

In $[1,2]$ for characterizing the consistency between the polarization states the laser object field in the points $\left(r_{1}, r_{2}\right)$ with the intensities $I\left(r_{1}\right), I\left(r_{2}\right)$ a new parameter-complex degree of mutual polarization (CDMP) in the next form

$$
V\left(r_{1}, r_{2}\right)=\frac{\left(U_{x}\left(r_{1}\right) U_{x}^{*}\left(r_{2}\right)+U_{y}\left(r_{1}\right) U_{y}^{*}\left(r_{2}\right)\right)^{2}}{I\left(r_{1}\right) I\left(r_{2}\right)} .
$$

This "two-point" theoretical approach was extended to the analysis of polarization-inhomogeneous laser images of human BT with the aim of experimental diagnostics of optical anisotropic structure [3]. In [4] a method of direct polarization measurement of the real part of CDMP for different points $\left(r_{1}, r_{2}\right)$ of optically thin image (attenuation coefficient $\tau \leq 0,1)$ of BT layers is proposed

$$
\begin{aligned}
& \operatorname{Re}\{V\} \\
& \equiv \widetilde{V}\left(r_{1}, r_{2}\right) \\
& =\frac{\left\{U_{x}\left(r_{1}\right) U_{x}\left(r_{2}\right)-U_{y}\left(r_{1}\right) U_{y}\left(r_{2}\right) \cos \left[\varphi\left(r_{1}\right)-\varphi\left(r_{2}\right)\right]\right\}^{2}}{I\left(r_{1}\right) I\left(r_{2}\right)},
\end{aligned}
$$

where $\varphi\left(r_{1}\right)$ and $\varphi\left(r_{2}\right)$ are the phase shifts between the orthogonal components $U_{x}, U_{y}$ of laser amplitude in object field.

The ranges of changes of the 1st-4th distribution order statistic moments $\tilde{V}(x, y)$ of the corresponding laser images, important for diagnostics of the human connective tissue oncologic state were determined in $[5,6]$. On the other hand, such analysis techniques of $\tilde{V}(x, y)$ lead to disregarding the BT extracellular matrix birefringence, which is a principal physical mechanism of their polarizationheterogeneous images formation [7-11]. That is why it appears to be important to search for new diagnostic parameters directly characterizing the degree of consistency of optical axes and birefringence orientations of various points of protein fibrils network forming the BT extracellular matrix [3].

This paper is aimed on investigation of diagnostic possibilities of optical-anisotropic structure of biological tissues extracellular matrix of different morphology and physiological state by means of statistic analysis of a new parameter-CDMA coordinate distribution. 


\section{Theoretical Analysis of Laser Radiation Parameters Transformation by the Network of Optically Uniaxial Birefringent Liquid Crystals}

The processes of laser beam interaction with $\mathrm{BT}$ are discussed in the following model approximation [12-16]:

(1) Morphologically all of variety of human tissues consists of four main types (connective, muscle, epithelial, and nervous), each of it is formed by a twocomponent amorphous-crystalline structure.

(2) The crystalline component of the BT (extracellular matrix) is formed by a network of coaxial cylindrical liquid crystals (collagen, elastin, myosin, etc, fibrils).

(3) From the optical point of view, protein fibrils possess properties of optically uniaxial crystals with the index of birefringence $\Delta n$. Parameters of anisotropy $(\rho(r)$, $\delta(r))$ of these fibrils in every point $(r)$ are described by the operator of Jones [17]

$$
\begin{aligned}
& \{D\}=\left\|\begin{array}{ll}
d_{11}(r) & d_{12}(r) \\
d_{21}(r) & d_{22}(r)
\end{array}\right\| \\
& =\left|\begin{array}{cc}
\cos ^{2} \rho(r)+\sin ^{2} \mathfrak{A} & \mathfrak{B} \\
\mathfrak{B} & \sin ^{2} \rho(r)+\cos ^{2} \mathfrak{A}
\end{array}\right|,
\end{aligned}
$$

where $\mathfrak{B}$ denotes $\cos \rho(r) \sin \rho(r)[1-\exp (-i \delta(r))]$ and $\mathfrak{A}$ denotes $\rho(r) \exp (-i \delta(r))$.

Here $\rho(r)$ - the angle (orientation) of the optical axis, which is determined by the direction of packing of birefringent $(\Delta n)$ protein fibrils with a transverse size $d(r)$; $\delta(r)=(2 \pi / \lambda) \Delta n d(r)$-phase shift of laser beam with the wavelength $\lambda$ at coordinate $r$.

It should be mentioned that further consideration of the possibilities of two-point Jones matrix description of complex anisotropy will be performed in a $2 \mathrm{D}$ approximation for $(x, y)$ plane of biological crystals anisotropic layer $-r \equiv$ $r(x, y)$.

With accordance to the approach outlined in [2], use (1)-(3) we obtain the following expression for the parameter $W\left(r_{1}, r_{2}\right)$ (complex degree of mutual anisotropy-CDMA) that characterizes the degree of coordination between the orientation $\left(\rho_{1}\left(r_{1}\right), \rho_{2}\left(r_{2}\right)\right)$ and phase $\left(\delta_{1}\left(r_{1}\right) ; \delta_{2}\left(r_{2}\right)\right)$ parameters of the anisotropy of different points $\left(r_{1}, r_{2}\right)$ in the plane of birefringent protein crystals network of the extracellular matrix

$$
W\left(r_{1}, r_{2}\right)=\frac{A\left(r_{1}, r_{2}\right)+B\left(r_{1}, r_{2}\right)}{C\left(r_{1}, r_{2}\right)},
$$

where

$$
\begin{aligned}
A=[ & \left.d_{11}\left(r_{1}\right)+d_{12}\left(r_{1}\right) \operatorname{tg} \Omega_{0} \exp \left(-i \varphi_{0}\right)\right] \\
\times & {\left[d_{11}\left(r_{2}\right)+d_{12}\left(r_{2}\right) \operatorname{tg} \Omega_{0} \exp \left(-i \varphi_{0}\right)\right]^{*}, } \\
B=\{ & {\left[d_{21}\left(r_{1}\right)+d_{22}\left(r_{1}\right) \operatorname{tg} \Omega_{0} \exp \left(-i \varphi_{0}\right)\right] } \\
& \left.\times\left[d_{21}\left(r_{2}\right)+d_{22}\left(r_{2}\right) \operatorname{tg} \Omega_{0} \exp \left(-i \varphi_{0}\right)\right]^{*}\right\} \\
\times & \exp \left\{-i\left[\delta_{2}\left(r_{2}\right)-\delta_{1}\left(r_{1}\right)\right]\right\}, \\
C=\{[ & \left.d_{11}\left(r_{1}\right)+d_{12}\left(r_{1}\right) \operatorname{tg} \Omega_{0} \exp \left(-i \varphi_{0}\right)\right] \\
& \times\left[d_{11}\left(r_{1}\right)+d_{12}\left(r_{1}\right) \operatorname{tg} \Omega_{0} \exp \left(-i \varphi_{0}\right)\right]^{*} \\
& +\left[d_{21}\left(r_{1}\right)+d_{22}\left(r_{1}\right) \operatorname{tg} \Omega_{0} \exp \left(-i \varphi_{0}\right)\right] \\
& \left.\times\left[d_{21}\left(r_{1}\right)+d_{22}\left(r_{1}\right) \operatorname{tg} \Omega_{0} \exp \left(-i \varphi_{0}\right)\right]^{*}\right\} \\
+ & \left\{\left[d_{11}\left(r_{2}\right)+d_{12}\left(r_{2}\right) \operatorname{tg} \Omega_{0} \exp \left(-i \varphi_{0}\right)\right]\right. \\
& \times\left[d_{11}\left(r_{2}\right)+d_{12}\left(r_{2}\right) \operatorname{tg} \Omega_{0} \exp \left(-i \varphi_{0}\right)\right]^{*} \\
& +\left[d_{21}\left(r_{2}\right)+d_{22}\left(r_{2}\right) \operatorname{tg} \Omega_{0} \exp \left(-i \varphi_{0}\right)\right] \\
& \left.\times\left[d_{21}\left(r_{2}\right)+d_{22}\left(r_{2}\right) \operatorname{tg} \Omega_{0} \exp \left(-i \varphi_{0}\right)\right]^{*}\right\} .
\end{aligned}
$$

Here $\varphi_{0}$ - (constant and crystals network anisotropic parameters independent) the phase shift between the orthogonal components $U_{0 x}$ and $U_{0 y}$ of the illuminating laser beam amplitude, $\operatorname{tg} \Omega_{0}=U_{0 y} / U_{0 x}$. Multipliers $\exp \left(-i \varphi_{0}\right)$ and $\operatorname{tg} \Omega_{0}$ are defined by illuminated laser beam polarization state and birefringent biological crystals optical-geometric structure independent.

Analysis of (4) and (5) shows the dependence of $W\left(r_{1}, r_{2}\right)$ on rotation of the polarization plane of the illuminating laser wave or rotation of the BT layer to the irradiation direction. The only exception is a case of irradiation of BT by the circular polarized $\left(\operatorname{tg} \Omega_{0}=1 ; \varphi_{0}=\right.$ $0,5 \pi)$ laser wave $U_{0}$

$$
U_{0}=\left(\begin{array}{c}
U_{0 x} \\
U_{0 y} \exp \left(-i \varphi_{0}\right)
\end{array}\right) \equiv \frac{1}{\sqrt{2}}\left(\begin{array}{l}
1 \\
i
\end{array}\right)
$$

In accordance with (6) the equations (4) and (5) become the following form:

$$
W\left(r_{1}, r_{2}\right)=\frac{\left\{\left[d_{11}\left(r_{1}\right)+i d_{12}\left(r_{1}\right)\right]\left[d_{11}\left(r_{2}\right)+i d_{12}\left(r_{2}\right)\right]^{*}+\left[d_{21}\left(r_{1}\right)+i d_{22}\left(r_{1}\right)\right]\left[d_{21}\left(r_{2}\right)+i d_{22}\left(r_{2}\right)\right]^{*}\right\}^{2}}{I\left(r_{1}\right) I\left(r_{2}\right)} .
$$


Here $I\left(r_{1}\right)$ and $I\left(r_{2}\right)$ are determined by relations

$$
\begin{aligned}
I\left(r_{1}\right)=\{ & \left\{\left[d_{11}\left(r_{1}\right)+i d_{12}\left(r_{1}\right)\right]\left[d_{11}\left(r_{1}\right)+i d_{12}\left(r_{1}\right)\right]^{*}\right. \\
& \left.+\left[d_{21}\left(r_{1}\right)+i d_{22}\left(r_{1}\right)\right]\left[d_{21}\left(r_{1}\right)+i d_{22}\left(r_{1}\right)\right]^{*}\right\}, \\
I\left(r_{2}\right)=\{ & {\left[d_{11}\left(r_{2}\right)+i d_{12}\left(r_{2}\right)\right]\left[d_{11}\left(r_{2}\right)+i d_{12}\left(r_{2}\right)\right]^{*} }
\end{aligned}
$$

$$
\left.+\left[d_{21}\left(r_{2}\right)+i d_{22}\left(r_{2}\right)\right]\left[d_{21}\left(r_{1}\right)+i d_{22}\left(r_{2}\right)\right]^{*}\right\} .
$$

Taking the real part $\left(\operatorname{Re}\left\{d_{i k}(r)\right\} \equiv \tilde{d}_{i k}(r)\right)$ of the Jones matrix elements (3), we obtain an expression for the real part of the CDMA $\left(\operatorname{Re}\left\{W\left(r_{1}, r_{2}\right)\right\} \equiv \widetilde{W}\left(r_{1}, r_{2}\right)\right)$ of extracellular matrix of BT layer, which can be obtained by direct polarization measurements

$$
\widetilde{W}\left(r_{1}, r_{2}\right)=\frac{\left\{\left[\tilde{d}_{11}\left(r_{1}\right) \tilde{d}_{11}\left(r_{2}\right)-\tilde{d}_{12}\left(r_{1}\right) \tilde{d}_{12}\left(r_{2}\right)\right]+\left[\tilde{d}_{21}\left(r_{1}\right) \tilde{d}_{21}\left(r_{2}\right)-\tilde{d}_{22}\left(r_{1}\right) \tilde{d}_{22}\left(r_{2}\right)\right]\right\}^{2}}{I\left(r_{1}\right) I\left(r_{2}\right)},
$$

where

$$
\begin{gathered}
\tilde{d}_{11}\left(r_{1,2}\right)=\cos ^{2} \rho\left(r_{1,2}\right)+\sin ^{2} \rho\left(r_{1,2}\right) \cos \delta\left(r_{1,2}\right), \\
\tilde{d}_{12,21}\left(r_{1,2}\right)=\cos \rho\left(r_{1,2}\right) \sin \rho\left(r_{1,2}\right)\left(1-\cos \delta\left(r_{1,2}\right)\right), \\
\tilde{d}_{22}\left(r_{1,2}\right)=\sin ^{2} \rho\left(r_{1,2}\right)+\cos ^{2} \rho\left(r_{1,2}\right) \cos \delta\left(r_{1,2}\right) .
\end{gathered}
$$

Let us analyze the relationship of CDMA $\widetilde{W}\left(r_{1}, r_{2}\right)$ of BT layer extracellular matrix with variations orientations $\rho_{1}\left(r_{1}\right)$, $\rho_{2}\left(r_{2}\right)$ of optical axes and the phase shifts $\delta_{1}\left(r_{1}\right), \delta_{2}\left(r_{2}\right)$ that introduced by the protein crystals of the extracellular matrix in the points $\left(r_{1}, r_{2}\right)$.

Table 1 shows the main characteristic values of CDMA $\widetilde{W}\left(r_{1}, r_{2}\right)$ in two arbitrary points $\left(r_{1}, r_{2}\right)$ of the extracellular matrix of BT layer.

From the obtained data one can see that CDMA has a wide range of value changes $\left(0,0 \leq \widetilde{W}\left(r_{1}, r_{2}\right) \leq 1,0\right)$ against to changes of orientation $(\rho(r))$ and phase $(\delta(r))$ anisotropic parameters in different points of biological crystals network. Therefore, the coordinate distribution $\widetilde{W}(x, y)$ can be used in diagnostics of optical-geometric structure of biological tissues layers.

\section{Optical Scheme and Experimental Measurements of Coordinate CDMA Distributions of Biological Tissue}

Experimental investigations were carried out in the classical polarimeter the main parts and elements of which are presented in Figure $1[4,5]$.

It was illuminated by collimated $\left(\varnothing=10^{4} \mu \mathrm{m}\right) \mathrm{He}$ Ne laser beam $(\lambda=0.6328 \mu \mathrm{m})$ with the power of $50 \mu \mathrm{W}$. Polarization illuminator (quarter-wavelength plates 3, 5 and polarizer 4) formed the beam with arbitrary polarization azimuth and ellipticity. Polarization images of BT by means of microobjective 7 (focal distance: $1.5 \mathrm{~cm}$, aperture: 0.2 , magnification: $4 \mathrm{x}$ ) were projected into the plane of lightsensitive area of CCD camera (overall amount of pixels: 800 $\times 600$, light sensitive area size: $4000 \times 3000 \mu \mathrm{m}$, deviation of photosensitive characteristics from linear no more then $15 \%)$, which provided the range of measuring the structural elements of BT with the resolution 2-2000 $\mu \mathrm{m}$. Maximal resolution verification $(2 \mu \mathrm{m})$ where performed using the stage micrometer (linear scale), which image was projected into the light sensitive area of CCD camera with the help of microobjective 7.

Minimal resolution $(2000 \mu \mathrm{m})$ corresponds to the situation when the light sensitive area of CCD camera is entirely filled by two equal sized structural elements (light and dark) of stage micrometer. The conditions of the experiment were chosen in such a way that it enabled to reduce the spaceangular aperture filtering while forming the BT images. This was ensured by conformance of angular characteristics of indicatrices of light scattering by the BT samples $\left(\Delta \gamma \approx 16^{0}\right)$ and angular aperture of microobjective $\left(\Delta \omega=20^{\circ}\right)$. Here $\Delta \gamma$ is the solid angle within which $98 \%$ of all the energy of lightscattered radiation is concentrated.

Initially, according to the classical technique given in [11], it was measured two-dimensional distribution

$$
\begin{aligned}
&((m \times n) \equiv\left(\begin{array}{ccc}
r_{11} & \cdots & r_{1 m} \\
\cdots & \cdots & \cdots \\
r_{n 1} & \cdots & r_{n m}
\end{array}\right) \\
& \equiv(x=1 \div m ; y=1 \div n)- \\
& \\
&\text { coordinates of CCD camera pixels })
\end{aligned}
$$

of the real parts of Jones matrix elements $\tilde{d}_{i k}(m \times n)$ of BT histological section.

Further rotation of the analyzer 9 transmission plane $(\Theta)$ in the limits $\Theta=0^{\circ} \div 180^{\circ}$ it is possible to determine minimum $\left(I_{\min }\right)$ and maximum $\left(I_{\max }\right)$ levels of intensity for each pixel of CCD-camera 10. Thus, the arrays of the intensity extreme values $I_{\min }(m \times n), I_{\max }(m \times n)$ of laser images of BT histological Section 6 and the corresponding angles of rotation $\Theta_{\min }(m \times n) \Leftrightarrow I_{\min }(m \times n)$ of the analyzer 9 can be obtained.

On the basis of the obtained data it is possible to calculate the coordinate distribution of azimuth $\alpha(m \times n)$ 


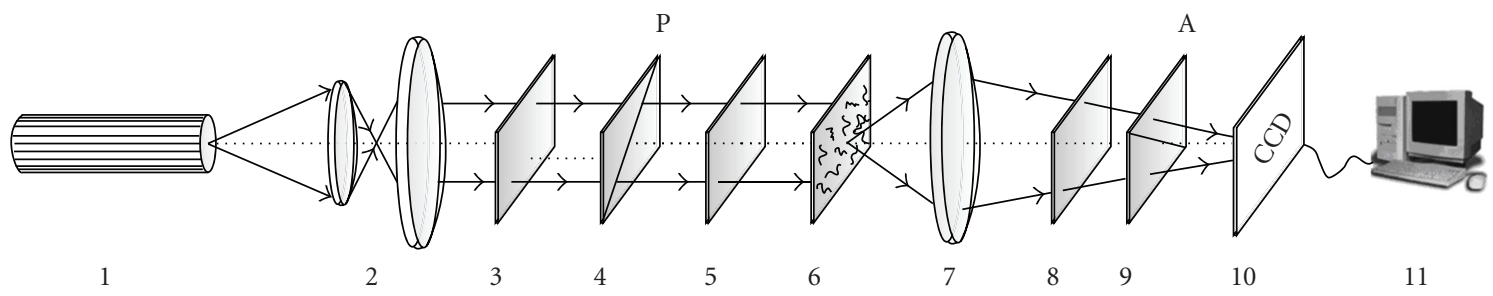

Figure 1: Optical scheme of polarimeter for measuring coordinate CDMA distributions of BT. Here $1-\mathrm{He}-\mathrm{Ne}$ laser $(\lambda=0.6328 \mu \mathrm{m}) ; 2-$ collimator; 3, 5 and 8-quarter-wave plates; 4 and 9 polarizers; 6-BT histological section; 7-projection microobjective; 10-CCD-camera; $11-\mathrm{PC}$.

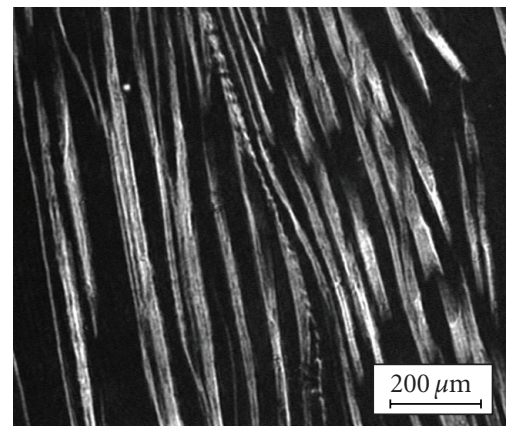

(a)

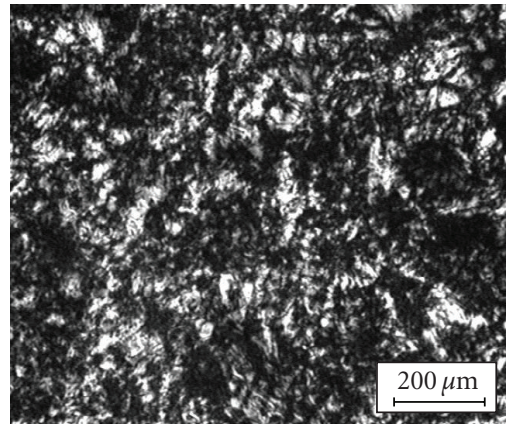

(b)

Figure 2: Architectonics of the muscular tissue (a) and the dermal layer (b) in crossed polarizer and analyzer.

TABLE 1: Characteristic values of CDMA $\widetilde{W}\left(r_{1}, r_{2}\right)$ of BT extracellular matrix.

\begin{tabular}{lcc}
\hline$\rho\left(r_{1}\right), \rho\left(r_{2}\right)$ & $\delta\left(r_{1}\right)=\delta\left(r_{2}\right)$ & $\widetilde{W}\left(r_{1}, r_{2}\right)$ \\
\hline$\rho\left(r_{1}\right)=\rho\left(r_{2}\right)$, & 0 & 1,0 \\
$\rho\left(r_{2}\right)=0,5 \pi+\rho\left(r_{2}\right)$ & $0,5 \pi$ & 1,0 \\
& $\pi$ & 1,0 \\
\hline & $\delta_{2}\left(r_{1}\right)=0,5 \pi+\delta_{1}\left(r_{2}\right)$ & $\widetilde{W}\left(r_{1}, r_{2}\right)$ \\
$\rho\left(r_{1}\right)=\rho\left(r_{2}\right)$, & $0,5 \pi$ & 0,5 \\
$\rho\left(r_{2}\right)=0,5 \pi+\rho\left(r_{2}\right)$ & $\pi$ & 0,5 \\
& $1,5 \pi$ & 0,5 \\
\hline & $\delta_{2}\left(r_{1}\right)=\pi+\delta_{1}\left(r_{2}\right)$ & $\widetilde{W}\left(r_{1}, r_{2}\right)$ \\
$\rho\left(r_{1}\right)=\rho\left(r_{2}\right)$, & $\pi$ & 0 \\
$\rho\left(r_{2}\right)=0,5 \pi+\rho\left(r_{2}\right)$ & $1,5 \pi$ & 0 \\
& $2 \pi$ & 0 \\
\hline
\end{tabular}

and ellipticity $\beta(m \times n)$ of polarization and determine the two-dimensional distribution of phase shifts by using the following algorithms

$$
\begin{gathered}
\alpha(m \times n)=0,5 \pi-\Theta(m \times n), \\
\beta(m \times n)=\operatorname{arctg} \frac{I_{\min }(m \times n)}{I_{\max }(m \times n)}, \\
\delta(m \times n)=\operatorname{arctg}\left[\frac{\operatorname{tg} 2 \beta(m \times n)}{\operatorname{tg} \alpha(m \times n)}\right] .
\end{gathered}
$$

The value of CDMA $\widetilde{W}\left(r_{1}, r_{2}=r_{1}+\Delta r\right)$ of the two points $\left(r_{1}, r_{1}+\Delta r\right)$ shifted by the interval $\Delta r$ of the network of protein crystals is calculated using the algorithm (9), (10). Coordinate distribution $\widetilde{W}(x, y)$ of the BT layer extracellular matrix is determined by scanning with the $\Delta r=1$ pix step in two mutually transverse directions $(x=1 \div m, y=1 \div n)$.

\section{Experimental Researches of Coordinate Distribution of CDMA of BT with Different Morphological and Physiological States}

It should be noted that the structure of the anisotropic component of the different types of BT is rather complicated and diverse [3]. Therefore the analysis of coordinate distribution of optical parameters that are characterized the BT extracellular matrix of various types objectively requires complex statistical, correlation, fractal and topological [11] and other approaches. In our paper we will restrict by study of the relationship of statistical moments of the 1st-4th order [12], which characterize the coordinate distribution $\widetilde{W}(x, y)$ of the BT extracellular matrix, with pathological changes of its orientation-phase structure.

As objects of study (Figure 2) were chosen the histological sections of myocardium muscle tissue (MT) (Figure 2(a)) and skin derma layer (SD) (Figure 2(b)). This choice objects of study due to the fact that birefringence indexes $(\Delta n)$ of myosin $\left(\mathrm{MT}-\Delta n=1,35 \cdot 10^{-2}\right.$ [13]) and collagen (SD: $\Delta n=1,5 \cdot 10^{-2}$ [14]) fibrils of the extracellular matrix of these tissues are similar. Therefore we can assume that the ranges of phase shifts values $(\delta(x, y))$ are determined 
TABLE 2: Statistic moments of the 1st-4th orders of CDMA $\widetilde{W}(x, y)$ and CDMP $\widetilde{V}(x, y)$ distributions of optically thin $(\tau=0,09)$ layers of physiologically normal and pathological changed samples of MT and SD.

\begin{tabular}{lcccccccc}
\hline BT & \multicolumn{9}{c}{ MT } & \multicolumn{4}{c}{ SD } \\
\hline \multirow{2}{*}{$M_{k}$} & \multicolumn{2}{c}{ Sound $(k=21)$} & \multicolumn{2}{c}{ Dystrophic changed $(k=19)$} & \multicolumn{2}{c}{ Sound $(k=20)$} & \multicolumn{2}{c}{ Oncological changed $(k=19)$} \\
& $\widetilde{W}$ & $\widetilde{V}$ & $\widetilde{W}$ & $\tilde{V}$ & $\widetilde{W}$ & $\widetilde{V}$ & $\widetilde{W}$ & $\tilde{V}$ \\
\hline$M_{1}$ & $0,8 \pm 0,09$ & $0,65 \pm 0,07$ & $0,58 \pm 0,06$ & $0,61 \pm 0,05$ & $0,38 \pm 0,04$ & $0,27 \pm 0,03$ & $0,33 \pm 0,04$ & $0,31 \pm 0,03$ \\
$M_{2}$ & $0,03 \pm 0,004$ & $0,04 \pm 0,006$ & $0,045 \pm 0,006$ & $0,043 \pm 0,005$ & $0,1 \pm 0,02$ & $0,07 \pm 0,008$ & $0,085 \pm 0,009$ & $0,063 \pm 0,008$ \\
$M_{3}$ & $18,1 \pm 1,92$ & $11,2 \pm 1,21$ & $6,54 \pm 0,87$ & $5,15 \pm 0,61$ & $9,4 \pm 0,87$ & $14,7 \pm 1,16$ & $21,7 \pm 1,96$ & $19,4 \pm 1,78$ \\
$M_{4}$ & $30,6 \pm 2,98$ & $41,8 \pm 3.17$ & $64,7 \pm 6,08$ & $61,9 \pm 5,87$ & $99,5 \pm 9,63$ & $76,9 \pm 6,98$ & $36,9 \pm 4,08$ & $42,6 \pm 4,17$ \\
\hline
\end{tabular}

TABLE 3: Statistic moments of the 1st-4th orders of CDMA $\widetilde{W}(x, y)$ and CDMP $\widetilde{V}(x, y)$ distributions of optically thick $(\tau=0,75)$ layers of physiologically normal and pathological changed samples of SD tissue.

\begin{tabular}{ccccccccc}
\hline BT & \multicolumn{4}{c}{ MT } & \multicolumn{4}{c}{ SD } \\
\hline \multirow{2}{*}{$M_{k}$} & \multicolumn{2}{c}{ Sound $(k=21)$} & \multicolumn{2}{c}{ Dystrophic changed $(k=19)$} & \multicolumn{2}{c}{ Sound $(k=20)$} & \multicolumn{2}{c}{ Oncological changed $(k=19)$} \\
& $\widetilde{W}$ & $\widetilde{V}$ & $\widetilde{W}$ & $\widetilde{V}$ & $\widetilde{W}$ & $\widetilde{V}$ & $\widetilde{W}$ & $\widetilde{V}$ \\
\hline$M_{1}$ & $0,57 \pm 0,062$ & $0,45 \pm 0,055$ & $0,48 \pm 0,042$ & $0,41 \pm 0,047$ & $0,45 \pm 0,051$ & $0,37 \pm 0,041$ & $0,63 \pm 0,057$ & $0,41 \pm 0,039$ \\
$M_{2}$ & $0,04 \pm 0,005$ & $0,05 \pm 0,006$ & $0,06 \pm 0,005$ & $0,065 \pm 0,007$ & $0,16 \pm 0,014$ & $0,27 \pm 0,033$ & $0,18 \pm 0,021$ & $0,23 \pm 0,025$ \\
$M_{3}$ & $12,7 \pm 1,15$ & $1,32 \pm 0,14$ & $6,34 \pm 0,71$ & $0,95 \pm 0,11$ & $8,65 \pm 0,83$ & $0,77 \pm 0,082$ & $12,1 \pm 1,18$ & $1,08 \pm 0,09$ \\
$M_{4}$ & $23,7 \pm 2,12$ & $1,78 \pm 0,98$ & $41,7 \pm 3,85$ & $1,29 \pm 0,16$ & $43,3 \pm 4,88$ & $1,13 \pm 0,15$ & $29,7 \pm 3,07$ & $0,86 \pm 0,09$ \\
\hline
\end{tabular}

primarily by transverse geometric dimensions $d(x, y)$ of protein crystals. Range of sizes for a network of SD collagen fibrilsconstitute the next interval $d=5 \mu \mathrm{m} \div 45 \mu \mathrm{m}$. For a MT layer of myosin fibrils the variations of sizes is in the range $d=25 \mu \mathrm{m} \div 35 \mu \mathrm{m}$.

Pathological changes of the MT morphological structure of (myocardial dystrophy) appear in the reduction of birefringence and disordering of packing direction of network myosin fibrils. The peculiarity of the extracellular matrix structure of sound SD is a random distribution of directions $(\rho(x, y))$ of the protein crystals optical axes (Figure 2). Extracellular matrix of oncological changed object of investigation is characterized by a set of ordered "the newly formed" collagen fibrils with a greater value of the birefringence parameter [16].

Therefore, comparative studies of CDMA coordinate distributions of such BT extracellular matrix will help to determine the range of statistical parameters variation of $\widetilde{W}(x, y)$ and on this basis, to implement the diagnostic of their physiological state.

The series of coordinate distributions $(x=1$ pix $\div$ 600 pix; $y=1$ pix $\div 800$ pix-fragments (a), (d); 50 pix $\times$ 50 pix-fragments (b), (e)) and the histograms (fragments (c), (f)) of CDMA values $\widetilde{W}(x, y)$ of physiologically normal (fragments (a), (b), (c)) and pathologically changed (fragments (d), (e), (f)) histological sections of MT (Figure 3) and SD (Figure 4) histological sections.

The analysis of the obtained experimental data discovered that the coordinate distributions of CDMA (Figures $3(\mathrm{a}), 3(\mathrm{~d}), 4(\mathrm{a})$, and $4(\mathrm{~d})$ are formed by the set of the local areas (domains $\widetilde{W} \approx$ const,-Figures $3(\mathrm{~b}), 3(\mathrm{e})$, and $4(\mathrm{~b})$, $4(\mathrm{e}))$ with the maximum possible range of variation $0,0 \leq$ $\widetilde{W} \leq 1,0$ (Figures $3(\mathrm{c}), 3(\mathrm{f})$ and $4(\mathrm{c}), 4(\mathrm{f})$ ).
Two-dimensional distribution (Figures 3(a) and 3(d)) of CDMA $\widetilde{W}(x, y)$ of MT samples are formed by the domains (Figure 3 (b) and $3(\mathrm{~d})$ ) with sizes $10 \mu \mathrm{m} \div 30 \mu \mathrm{m}$. Degenerative changes of myosin fibrils network appears in the process of the scale reducing $(15 \%-30 \%)$ of the domain structure of CDMA coordinate distribution.

Coordinate structure of CDMA parameter $\widetilde{W}(x, y)$ of the SD collagen network is smaller-scale (Figures $4(\mathrm{a}), 4(\mathrm{~d})$ ). The range of geometric dimensions of domain changes (Figures 4 (b) and $4(\mathrm{~d})$ ) varies in limits $5 \mu \mathrm{m} \div 15 \mu \mathrm{m}$. Cancer changes of SD architectonic network are revealed in some increase (up to $10 \%-15 \%$ ) of the local domains scale.

The experimental data satisfactorily correlate with the proposed model analysis of the extracellular matrix structure with the help of CDMA. Thus, there will always be points $(r, r+\Delta r)$ in the network of protein fibrils that define such relationships between the parameters of anisotropy $(\rho, \delta)$ (Table 1), which correspond to extreme values of CDMA

$$
\widetilde{W}(r, r+\Delta r)=\left\{\begin{array}{l}
0 ; \\
1,0 .
\end{array}\right.
$$

On the other hand, the specificity of the morphological construction of BT extracellular matrix of various types is manifested in different probabilities of CDMA $\widetilde{W}(x, y)$ values. It is believed that for directional ordered $(\rho(r=$ $x, y)$ ) and the geometric dimensions $(d(r=x, y))$ of MT myosin fibrils network (Figure 3(a)) the dispersion of the orientations of optical axes and the phase shifts are substantially less than for the disordered network of SD collagen fibrils (Figure 4(a)). Therefore, the distribution of CDMA random values of MT layer predominantly localized in a small range $(0,5 \leq \widetilde{W} \leq 1,0)$ relatively to the main 


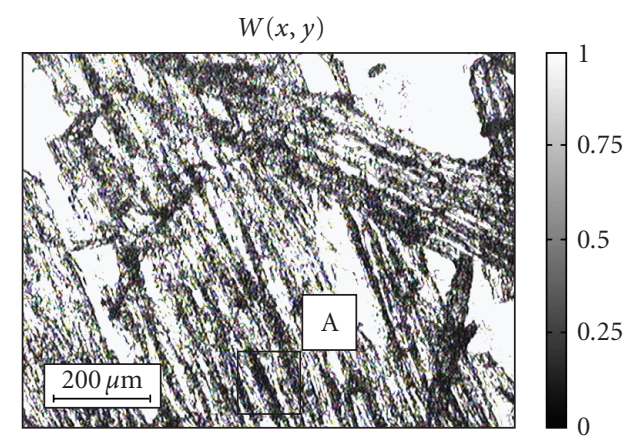

(a)

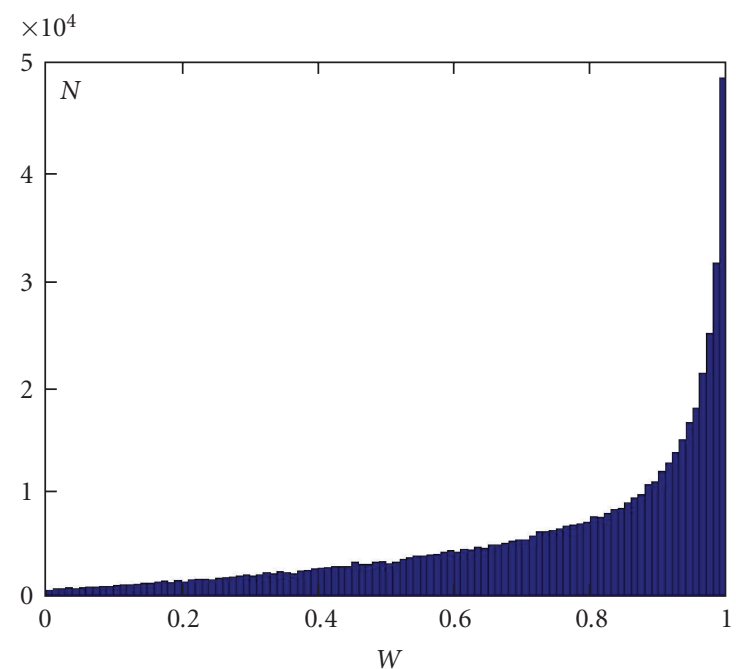

(c)

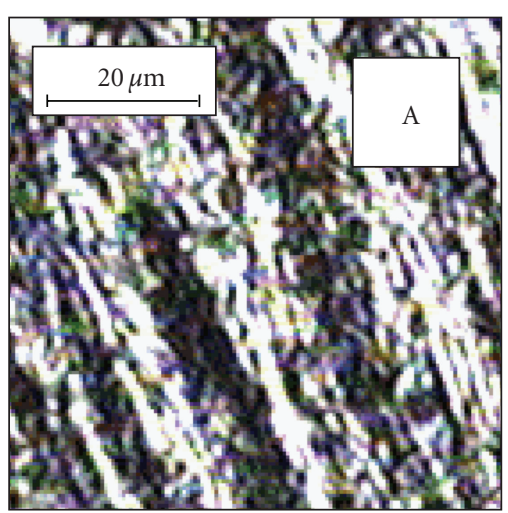

(b)

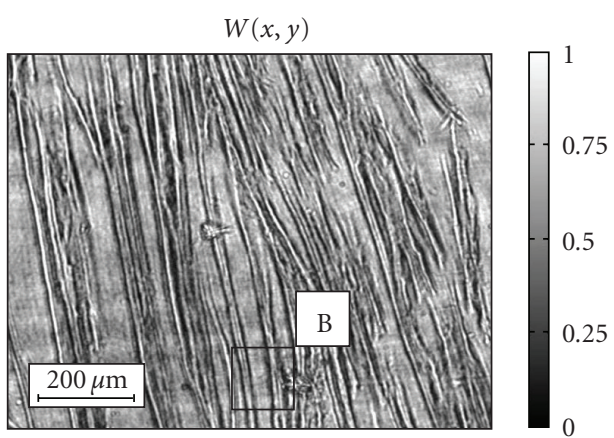

(d)

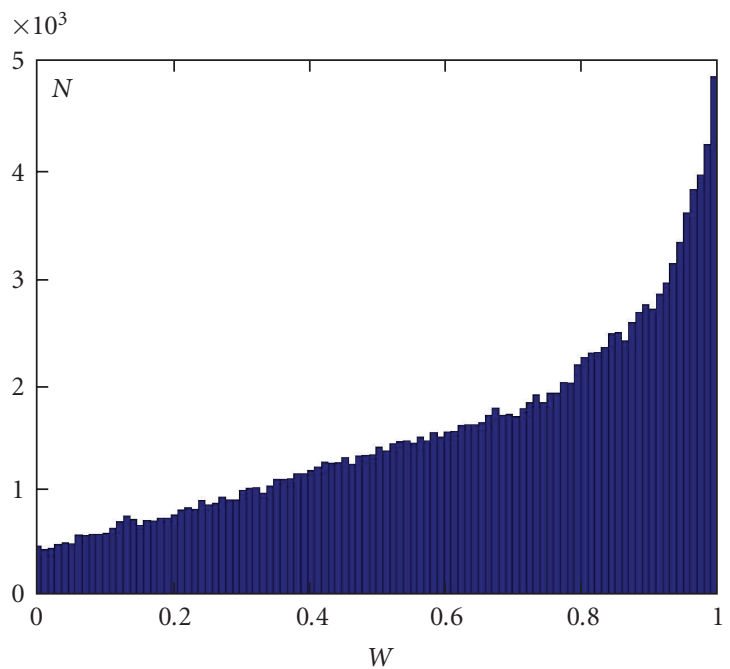

(f)

FIgURE 3: Coordinate distributions ( 600 pix $\times 800$ pix -fragments a, d; 50 pix $\times 50$ pix -fragments b, e) and histograms (fragments c, f) of values $\widetilde{W}(x, y)$ of physiologically normal (a, b, c) and pathologically changed (d, e, f) of MT. 


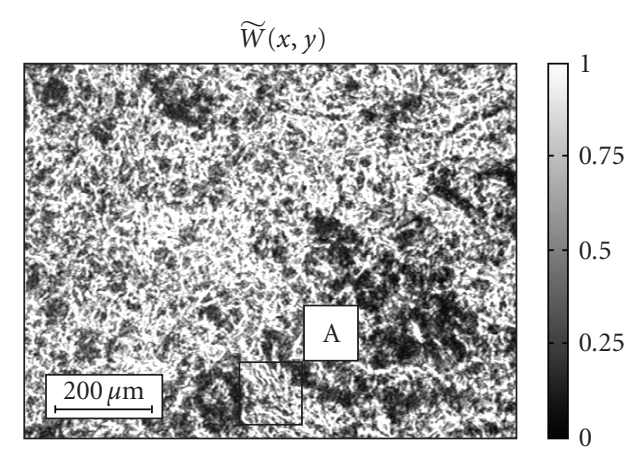

(a)

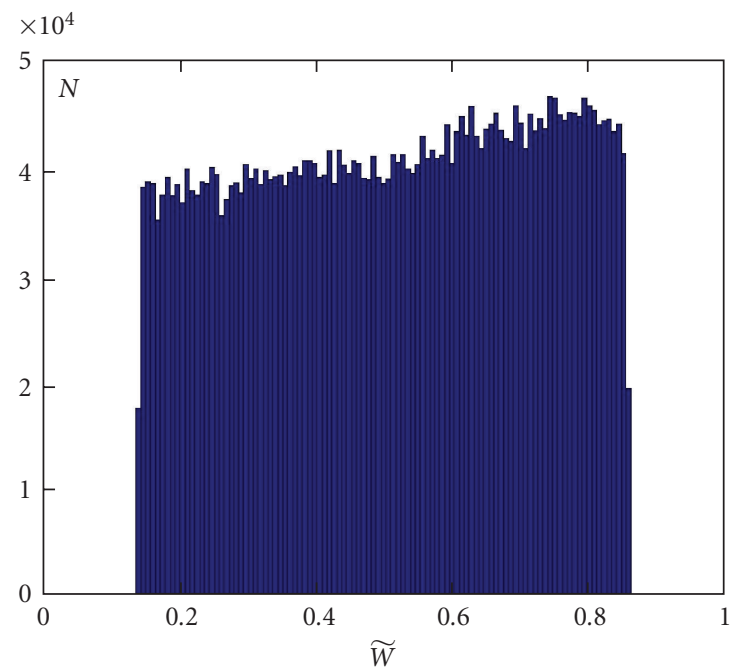

(c)

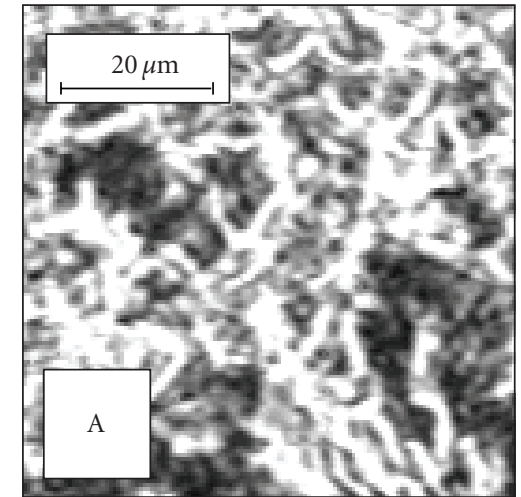

(b)

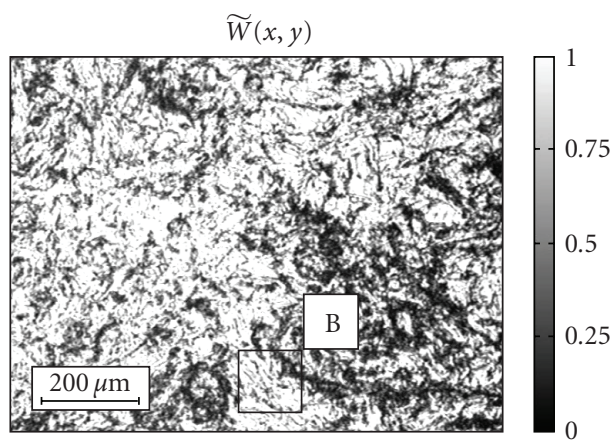

(d)

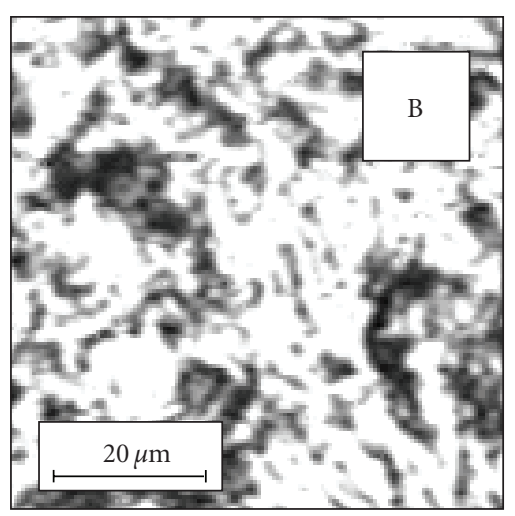

(e)

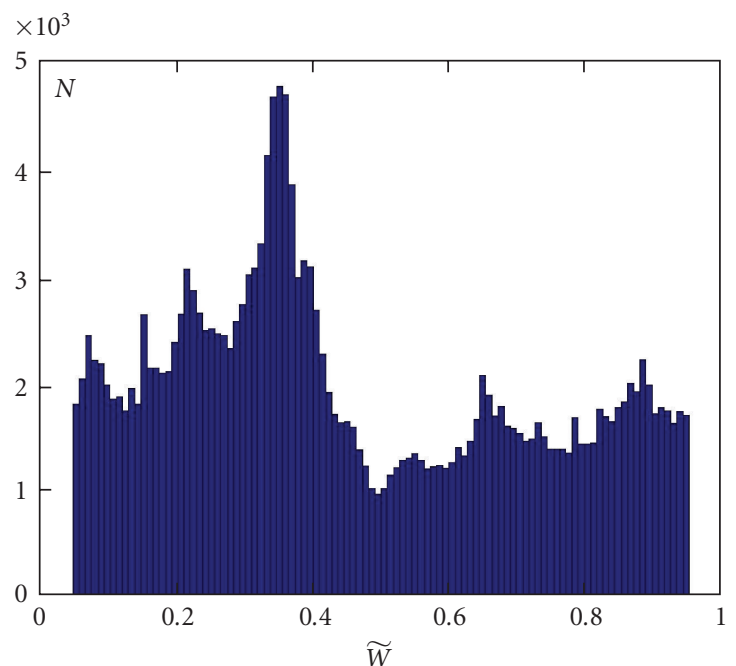

(f)

FIGURE 4: Coordinate distributions ( 600 pix $\times 800$ pix — fragments a, d; 50 pix $\times 50$ pix-fragments b, e) and histograms (fragments c, f) of values $\widetilde{W}(x, y)$ of physiologically normal $(\mathrm{a}, \mathrm{b}, \mathrm{c})$ and oncological changed $(\mathrm{d}, \mathrm{e}, \mathrm{f})$ of SD tissue. 
extrema (Figure 3(a)). Histograms of CDMA values $\widetilde{W}$ of SD layer are fairly equiprobable distribution in the broadest possible range $(0 \leq \widetilde{W} \leq 1,0)$ of this parameter changing (Figure 4(a)).

Degenerative changes of MT myosin crystal network are shown (Figure 3(e)) in the distribution of extreme values CDMA $(\widetilde{W} \rightarrow 1,0)$ to lower values $(\widetilde{W}=0,6 \div 0,8)$. For chaotically oriented network of collagen fibrils of the sound tissue extracellular matrix (Figures $4(\mathrm{a})$ and $4(\mathrm{~b})$ ) the values of $\widetilde{W}(x, y)$ histograms represent rather equiprobable distributions (Figure 4(c)). Early oncologic changes of SD are accompanied with the formation of the collagen fibrils growth direction. It is optically shown (Figures 4(d) and $4(\mathrm{e}))$ in some localization of the CDMA random values distribution (Figure $4(\mathrm{f})$ ) in the domain of $\widetilde{W}=0,4 \div 0,6$ extrema.

In order to obtain objective criteria of diagnostic efficiency, the comparative investigation of $\operatorname{CDMP}(\tilde{V}(x, y))$ $[4,5]$ and CDMA $\widetilde{W}(x, y)$ techniques was performed in the conditions of single and multiple scattering of laser radiation by the layers of MT and SD.

\section{Comparative Statistical Analysis of Coordinate Distributions of CDMA of BT Extracellular Matrix and CDMP of Its Laser Images}

With the aim of determination the ranges of statistical moments changes that characterize the coordinate distribution of $\widetilde{W}(x, y)$ and $\widetilde{V}(x, y)$ four groups of histological sections of MT (sound: 21 samples; dystrophic changed: 19 samples) and SD layer samples were formed (sound: 20 samples; oncological changed: 19 samples).

Statistical moments of the 1st-4th-order $M_{k=1,2,3,4}$ of

$$
Q=\left\{\begin{array}{l}
\tilde{V} \\
\widetilde{W}
\end{array}\right.
$$

distributions were calculated with the help of Matlab software product on the basis of the following algorithms [12]

$$
\begin{array}{cc}
M_{1}=\frac{1}{N} \sum_{i=1}^{N}\left|Q_{i}\right|, & M_{2}=\sqrt{\frac{1}{N} \sum_{i=1}^{N} Q_{i}^{2},} \\
M_{3}=\frac{1}{M_{2}^{3}} \frac{1}{N} \sum_{i=1}^{N} Q_{i}^{3}, & M_{4}=\frac{1}{M_{2}^{2}} \frac{1}{N} \sum_{i=1}^{N} Q_{i}^{4},
\end{array}
$$

where $N=m \times n$-quantity of pixels of CCD-camera photosensitive area.

In Tables 2 and 3 the comparative results of calculations of the average $\left(M_{1}\right)$, dispersion $\left(M_{2}\right)$, the skewness $\left(M_{3}\right)$ and the kurtosis $\left(M_{4}\right)$ coefficients of CDMA $\widetilde{W}(x, y)$ distributions of four groups of MT and SD of different optical thickness (attenuation coefficient $\tau=0,09$ and $\tau=0,75$ ) and of CDMP $\widetilde{V}(x, y)$ of their laser images are presented.
From the obtained data about the coordinate distributions of CDMA of optically thin $(\tau=0,09)$ layers of MT and $\mathrm{SD}$ tissue one can see the following.

(i) The average and dispersion of distributions $\widetilde{W}(x, y)$ of physiologically normal and pathological changed MT and SD differ insufficiently within $1.3\left(M_{1}\right)-$ $1.5\left(M_{2}\right)$ times. For two-dimensional distributions $\tilde{V}(x, y)$ of laser images there is practically no difference between $M_{1}$ and $M_{2}$.

(ii) The skewness values $M_{3}$ of distributions $\widetilde{W}(x, y)$ of the investigated samples differ by 2.1 times; the kurtosis values-by 3.2 times. For CDMP distributions $\tilde{V}(x, y)$ the values of the 3 rd and 4th statistic moments vary for $M_{3}$-by 1.3 times; for $M_{4}$ - by 1.8 times.

On the other hand, with increasing the light scattering the distribution of azimuths and ellipticities of polarization of the BT object field are changed $[8-10,18,19]$. As a result, the interconnection between the parameters $\widetilde{W}\left(r_{1}, r_{2}\right)$ and $\tilde{V}\left(r_{1}, r_{2}\right)$ is destroyed (Table 3 ):

(i) As diagnostically actual parameters of the physiological state of optically thick BT the statistical moments of higher order can be used. Thus, skewness values $M_{3}$ of coordinate distributions $\widetilde{W}(x, y)$ for the sound and oncological changed SD tissue differ by 1.4 times; the kurtosis values $M_{4}$-by 1.45 times.

(ii) The values of the 3rd-4th order statistic moments of distributions $\widetilde{V}(x, y)$ of CDMP of object field of optically thick BT layers do not depend much on their physiological state and are the an order less than the values of CDMP $\widetilde{W}(x, y)$ skewness and the kurtosis.

\section{Conclusions}

To characterize the degree of consistency of parameters of the optically uniaxial birefringent (liquid crystals) protein fibrils nets of biological tissues a new parameter-complex degree of mutual anisotropy is suggested. The technique of polarization measuring the coordinate distributions of the complex degree of mutual anisotropy of biological tissues is developed. It is shown that statistic approach to the analysis of complex degree of mutual anisotropy distributions of biological tissues of various morphological and physiological states and optical thicknesses appears to be more sensitive and efficient in differentiation of physiological state in comparison with investigations of complex degree of mutual polarization of the corresponding laser images.

\section{References}

[1] M. Mujat, A. Dogariu, and G. S. Agarwal, "Interferometric measurement of the degree of polarization and control of the contrast of intensity fluctuations," Optics Letters, vol. 29, no. 13, pp. 1539-1541, 2004.

[2] J. Ellis and A. Dogariu, "Complex degree of mutual polarization,” Optics Letters, vol. 29, no. 6, pp. 536-538, 2004. 
[3] S. C. Cowin, "How is a tissue built?" Journal of Biomechanical Engineering, vol. 122, no. 6, pp. 553-569, 2000.

[4] O. V. Angelsky, A. G. Ushenko, and Y. G. Ushenko, "Complex degree of mutual polarization of biological tissue coherent images for the diagnostics of their physiological state," Journal of Biomedical Optics, vol. 10, no. 6, Article ID 060502, 2005.

[5] Y. G. Ushenko, "Complex degree of mutual polarization of Biotissue's Speckle-images," Ukrainian Journal of Physics, vol. 6, pp. 104-113, 2005.

[6] M. Mujat and A. Dogariu, "Polarimetric and spectral changes in random electromagnetic fields," Optics Letters, vol. 28, no. 22, pp. 2153-2155, 2003.

[7] O. V. Angelsky, A. G. Ushenko, V. P. Pishak, et al., "Polarizingcorrelative processing of images of statistic objects in the problem of visualization and topology reconstruction of their phase heterogeneity," in Photonics, Devices, and Systems, vol. 4016 of Proceedings of SPIE, pp. 419-424, 1999.

[8] S. P. Morgan, M. P. Khong, and M. G. Somekh, "Effects of polarization state and scatterer concentration on optical imaging through scattering media," Applied Optics, vol. 36, no. 7, pp. 1560-1565, 1997.

[9] S. G. Demos and R. R. Alfano, "Optical polarization imaging," Applied Optics, vol. 36, no. 1, pp. 150-155, 1997.

[10] S. L. Jacques, J. R. Roman, and K. Lee, "Imaging superficial tissues with polarized light," Lasers in Surgery \& Medicine, vol. 26, no. 2, pp. 119-129, 2000.

[11] A.G. Ushenko and V. P. Pishak, Coherent-Domain Optical Methods, Biomedical Diagnostics, Environmental and Material Science, Kluwer Academic Publishers, Dordrecht, The Netherlands, 2004.

[12] Y. A. Ushenko, "Statistical structure of polarizationinhomogeneous images of biotissues with different morphological structures," Ukrainian Journal of Physics, vol. 6, pp. 63-70, 2005.

[13] O. V. Angelsky, D. N. Burkovets, V. P. Pishak, and Y. A. Ushenko, "Polarization-correlation investigations of biotissue multifractal structures and their pathological changes diagnostics," Laser Physics, vol. 10, pp. 1136-1142, 2000.

[14] A. G. Ushenko, "The vector structure of laser biospeckle fields and polarization diagnostics of collagen skin structures," Laser Physics, vol. 10, no. 5, pp. 1143-1149, 2000.

[15] O. V. Angelsky, P. P. Maksimyak, and T. O. Perun, "Optical correlation method for measuring spatial complexity in optical fields," Optics Letters, vol. 18, no. 2, pp. 90-92, 1993.

[16] O. V. Angel'skiı̌, A. G. Ushenko, S. B. Ermolenko, et al., "Polarization-based visualization of multifractal structures for the diagnostics of pathological changes in biological tissues," Optics and Spectroscopy, vol. 89, no. 5, pp. 799-804, 2000.

[17] M. Born and E. Wolf, Principles of Optics, Cambridge University Press, Cambridge, UK, 1999.

[18] R. R. Alfano and J. G. Fujimoto, Advances in Optical Imaging and Photon Migration, vol. 2 of Topics in Optics and Photonics Series, Optical Society of America, Washington, DC, USA, 1996.

[19] S. Bartel and A. H. Hielscher, "Monte Carlo simulations of the diffuse backscattering Mueller matrix for highly scattering media," Applied Optics, vol. 39, no. 10, pp. 1580-1588, 2000. 

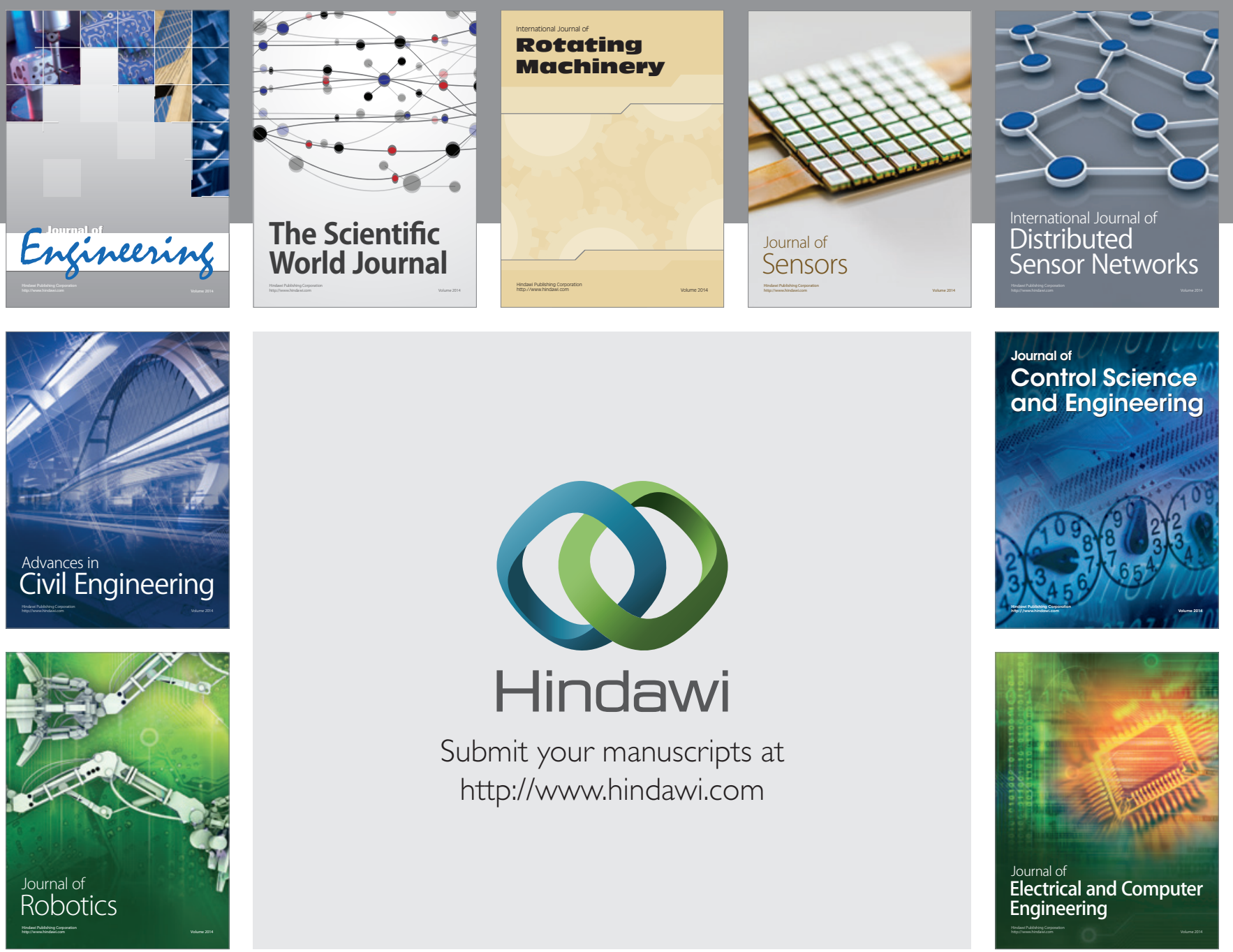

Submit your manuscripts at

http://www.hindawi.com
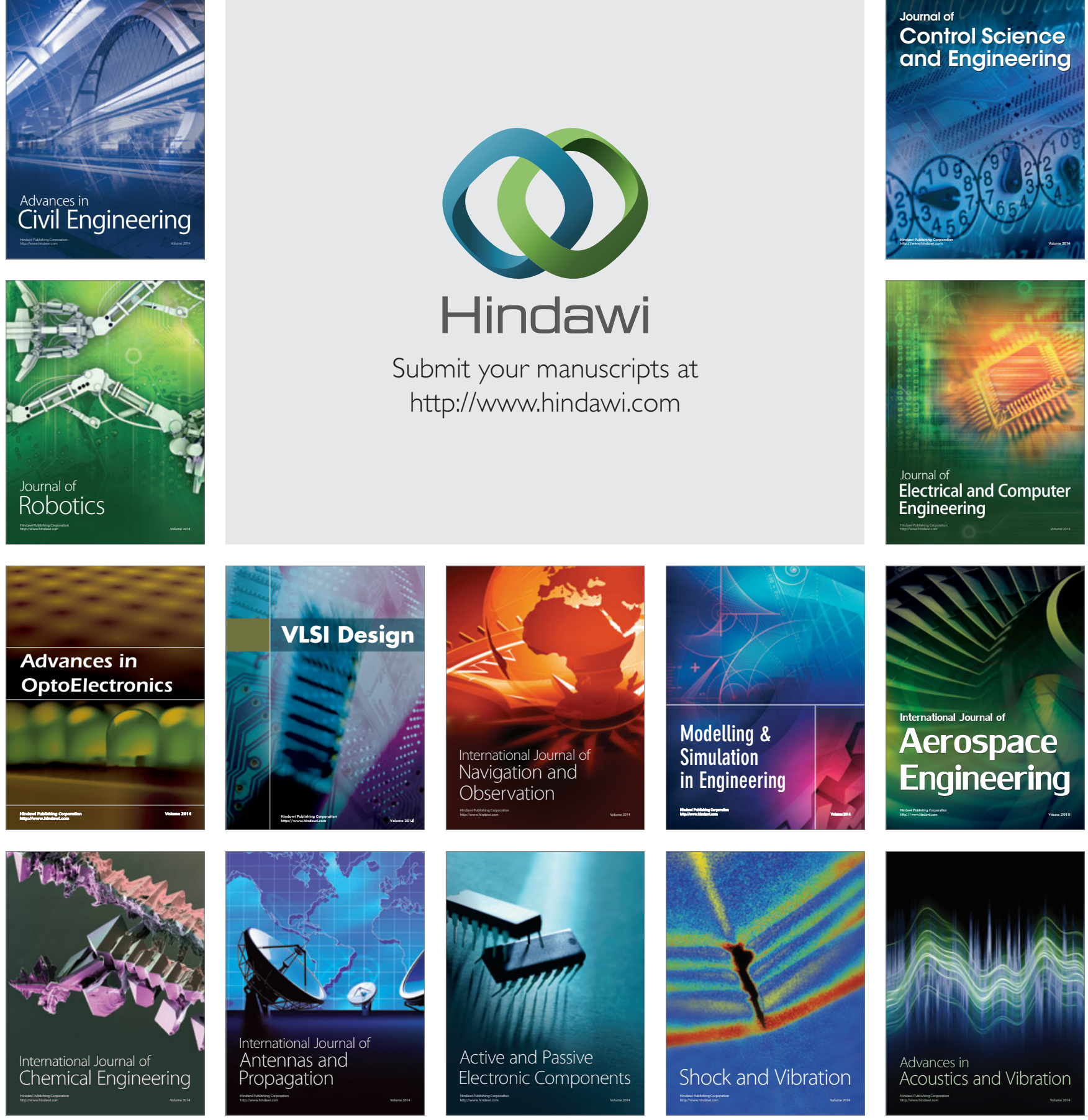